\title{
Neutron Interferometry at the National Institute of Standards and Technology
}

\author{
D. A. Pushin, ${ }^{1,2}$ M. G. Huber, ${ }^{3}$ M. Arif, ${ }^{3}$ C. B. Shahi, ${ }^{4}$ J. Nsofini, ${ }^{1,2}$ C. J. Wood, ${ }^{1,2}$ \\ D. Sarenac, ${ }^{1,2}$ and D. G. Cory ${ }^{1,5,6}$ \\ ${ }^{1}$ Institute for Quantum Computing, University of Waterloo, Waterloo, ON, Canada N2L 3G1 \\ ${ }^{2}$ Department of Physics and Astronomy, University of Waterloo, Waterloo, ON, Canada N2L 3G1 \\ ${ }^{3}$ National Institute of Standards and Technology, Gaithersburg, MD 20899, USA \\ ${ }^{4}$ Physics and Engineering Physics Department, Tulane University, New Orleans, LA 70118, USA \\ ${ }^{5}$ Department of Chemistry, University of Waterloo, Waterloo, ON, Canada N2L $3 G 1$ \\ ${ }^{6}$ Perimeter Institute for Theoretical Physics, Waterloo, ON, Canada N2L 2 Y5
}

Correspondence should be addressed to D. A. Pushin; dmitry.pushin@uwaterloo.ca

Received 30 July 2014; Accepted 19 August 2014

Academic Editor: Guillaume Pignol

Copyright (c) 2015 D. A. Pushin et al. This is an open access article distributed under the Creative Commons Attribution License, which permits unrestricted use, distribution, and reproduction in any medium, provided the original work is properly cited. The publication of this article was funded by SCOAP S $^{3}$

\begin{abstract}
Neutron interferometry has proved to be a very precise technique for measuring the quantum mechanical phase of a neutron caused by a potential energy difference between two spatially separated neutron paths inside interferometer. The path length inside the interferometer can be many centimeters (and many centimeters apart) making it very practical to study a variety of samples, fields, potentials, and other macroscopic medium and quantum effects. The precision of neutron interferometry comes at a cost; neutron interferometers are very susceptible to environmental noise that is typically mitigated with large, active isolated enclosures. With recent advances in quantum information processing especially quantum error correction (QEC) codes we were able to demonstrate a neutron interferometer that is insensitive to vibrational noise. A facility at NIST's Center for Neutron Research (NCNR) has just been commissioned with higher neutron flux than the NCNR's older interferometer setup. This new facility is based on QEC neutron interferometer, thus improving the accessibility of neutron interferometry to the greater scientific community and expanding its applications to quantum computing, gravity, and material research.
\end{abstract}

\section{Introduction}

The first single crystal neutron interferometer of Mach-Zehnder type was demonstrated by Rauch et al. in 1974 [1]. A few months later Colella, Overhauser, and Werner studied the effects of gravity on a neutron by tilting the interferometer and observing a gravity induced phase shift [2]. In subsequent years the experiment was improved and repeated several times with thorough studies of systematic uncertainties (such as Sagnac effect and crystallographic stress [3-6]). In recent years there has been renewed interest in use of single crystal neutron interferometry to study gravity $[7,8]$ and also in proposals for searching for non-Newtonian gravity [9], dark energy $[10,11]$, and other forces [12].

\section{Neutron Interferometer and Optics Facility at NIST}

The Neutron Interferometer and Optics Facility (NIOF) at NIST was built during the construction of the guide hall with vibration isolation in mind. It is placed on a separate foundation from the guide hall floor and consists of two vibration isolation stages. The first stage is a $40,000 \mathrm{~kg}$ table suspended on air-springs and controlled via a computer with micrometer level position. For a more detailed description of the system see [13]. The second vibration isolation stage consists of a $1,000 \mathrm{~kg}$ table and is not currently in use. The facility also has sound dumping panels and a temperature controlled enclosure around interferometer. Temperature is 
stabilized to $5 \mathrm{mK}$ precision at room temperature in order to improve the phase stability of the system [14]. When the temperature is stabilized at this level the interferometer phase is stable within a couple of degrees over a onemonth time scale. Neutrons are extracted from the cold guide and redirected to the interferometer enclosure via two sets of pyrolytic graphite (PG) crystal monochromators. The interferometer facility has access to a wide range of neutron wavelengths from $0.2 \mathrm{~nm}$ to $0.45 \mathrm{~nm}$. Neutrons in this facility can be polarized via a $\mathrm{V}$-shaped transmission supermirror polarizer with $93 \%$ polarization efficiency for $0.235 \mathrm{~nm}$ wavelength neutrons [15].

\section{Equivalence of a Neutron Interferometer and Light Mach-Zehnder Interferometer}

In the past years we have used several neutron interferometer designs that are equivalent to Mach-Zehnder optical interferometers (Figure 2). The first blade of the interferometer coherently splits the neutron beam into two paths (upper and lower) via Bragg diffraction, as illustrated in Figure 2(a). The middle blade(s) work as mirrors. They redirect and focus neutron paths into the third blade. At the third blade the neutron paths are recombined, and the resulting interference effects can be observed by ${ }^{3} \mathrm{He}$-detectors placed in both paths behind interferometer. Each neutron inside the interferometer (satisfying the Bragg conditions) can be described as the superposition of the two paths (i.e., "upper" and "lower"):

$$
\left.\left.\Psi=e^{i \phi_{1}} C_{1} \mid \text { upper }\right\rangle+e^{i \phi_{2}} C_{2} \mid \text { lower }\right\rangle,
$$

where $C_{1}$ and $C_{2}$ are normalization coefficients that depend on the reflection and transmission coefficients of the interferometer blades and $\phi_{1}$ and $\phi_{2}$ are phases that the neutron acquires passing through different paths. An important point is that the reactor flux is so low that there will be only one neutron inside the interferometer at a time. Thus we only observe neutron self-interference, and neutron-neutron interferences can be neglected. Several high contrast (>75\%) perfect Si crystal neutron interferometers are shown in Figure 2(b). They are on the order of $10 \mathrm{~cm}$ in size and so allow for the placement of an object (such as a sample, phase rotator, spin flipper, and neutron absorber) in one path without affecting the other path. This allows for the observation of interference effects due to neutrons passing through the interferometer with one path modified by this object [16]. This is why neutron interferometry is a clear example of macroscopic quantum coherence and a convenient test bed to study many aspects of quantum mechanics, neutron interactions, quantum information processing (QIP), and fundamental physics.

\section{Coherence and QIP}

The single crystal neutron interferometer is an ideal tool for the study of neutron coherence length. The clear way of changing the optical path length and shifting one path with respect to the other $[14,17-20]$ allows direct measurement of coherence lengths. The coherence length $\left(l_{c}\right)$ often depends on the momentum distribution $(\delta k)$ of neutrons entering the interferometer, and it is limited by Heisenberg's uncertainty relation

$$
l_{c}=\frac{1}{2 \delta k}
$$

To understand this limit in the measurement of the vertical coherence length we have engineered different momentum distributions using the focusing monochromator and set of slits as shown in Figure 3(a). By shielding some of the PG blades of the focusing monochromator we were able to create different vertical momentum distributions for the neutrons.

By measuring the contrast of the neutron interferometer we can directly map out the coherence function. In order to measure contrast we usually place fused silica flat (phase flag) in both neutron paths. By rotating this phase flag around vertical axis we modify the optical path length of neutrons traveling through different interferometer paths. This allows oscillations of neutron intensity $(I)$ on the detectors behind the interferometer. The contrast is defined as the ratio:

$$
\text { Contrast }=\frac{\max (I)-\min (I)}{\max (I)+\min (I)} .
$$

The dependence of the contrast on the vertical path separation created by prisms inside neutron interferometer [14] with different initial momentum distributions is shown in Figure 3(b) (from top: 9 blades, 5 blades, and 1 blade of the focusing monochromator). The red dots are measured data while straight lines are obtained from the measured momentum distributions. Hence by shaping the momentum distribution we can, in principle, achieve a desired coherence length. In this measurement the neutron wavelength was $0.271 \mathrm{~nm}$ while the highest obtained vertical coherence length was $79 \mathrm{~nm}$. Another important aspect to note is that the neutron path length is about $0.1 \times 10^{9} \mathrm{~nm}$ and is about $10^{9}$ bigger than the neutron wavelength. That is why the neutron interferometer is an extremely sensitive device. It is also very sensitive to external disturbances such as vibrational noise, temperature instabilities and gradients, and sounds. Neutrons used for interferometry have velocities on the order $1000 \mathrm{~m} / \mathrm{s}$ (which is relatively slow compared to the speed of light); that is why neutron interferometers are sensitive to low frequency vibrations. The setup shown in Figure 1 is designed to remove most of such disturbances and preserve long-time phase stability. However the massive vibration isolation stage keeps the interferometer further away from the source and makes it difficult to operate. As a result the facility has a low neutron fluency and is not particularly user friendly which is why neutron interferometers are rarely used for condensed matter applications.

To further help us deal with noise we can employ techniques from quantum information processing such as quantum error correction (QEC) schemes. One approach to passive QEC (well described by Lidar and Whaley in [21]) is a decoherence-free subspace (DFS), which corresponds to finding a subspace of a larger quantum system that is not affected by a noise process and then encoding our desired quantum state in this subspace. A DFS can be implemented in a neutron interferometer to make it insensitive to low 


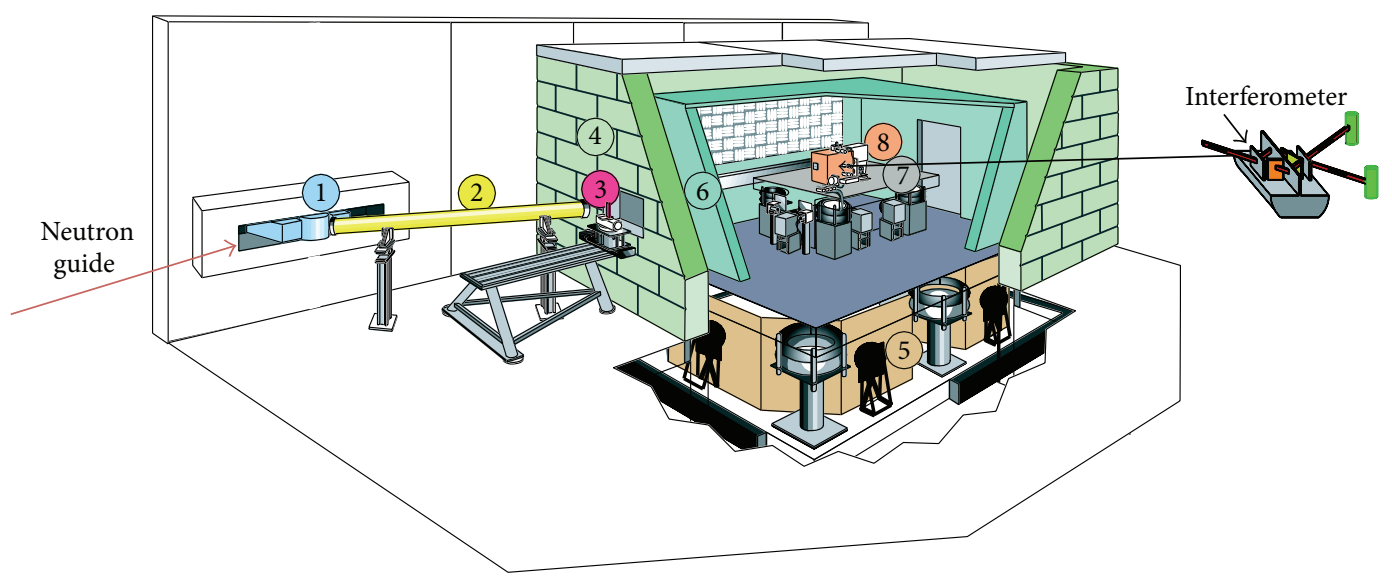
Components:
(1) Collimator/shutter
(5) Primary vibration isolation stage
(2) Helium-filled beam transport tube
6) Acoustic and thermal isolation enclosure
(3) Focusing pyrolytic graphite monochromator
(7) Secondary vibration isolation stage
(4) Outer environmental enclosure
(8) Enclosure for interferometer and detectors

FIGURE 1: Schematic of the Neutron Interferometry and Optics Facility for Neutron Research at the National Institute of Standards and Technology. The isolated 40,000 kg room is supported by six air-springs. The system uses active vibration control to eliminate vibrations above $0.5 \mathrm{~Hz}$. The temperature of the interferometer enclosure is stabilized to $5 \mathrm{mK}$ precision to improve long-term phase stability (diagram also appeared in [16]).

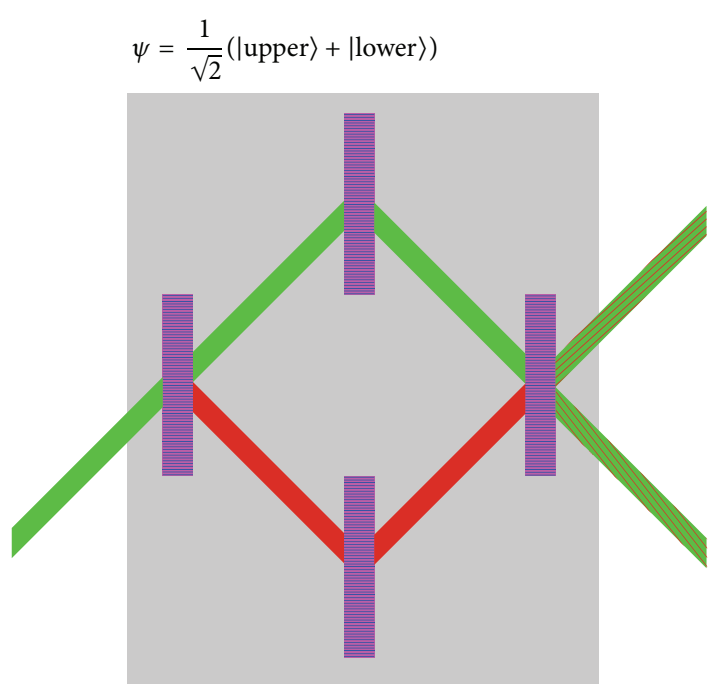

(a)

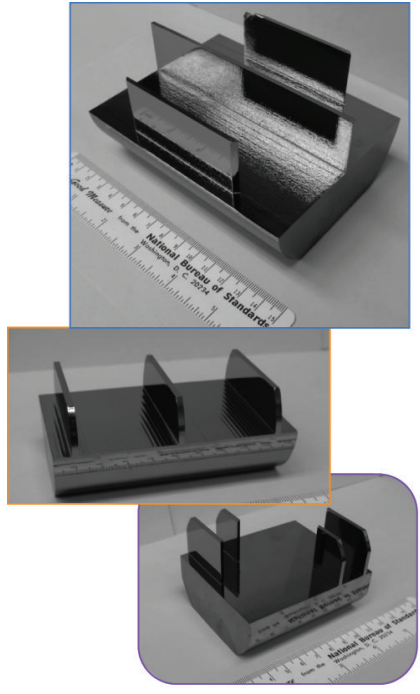

(b)

Figure 2: (a) Schematic diagram of the neutron paths in a perfect single crystal neutron interferometer. The neutron passing through the interferometer is coherently split into two paths (upper and lower) by the first interferometer blade, redirected by the middle blades (these blades represent mirrors of the light Mach-Zehnder interferometer and are recombined to observe interference effects by the last blade). The neutron's wave function can be described as superposition of the paths inside the interferometer. (b) Pictures of NIST interferometers with high contrast $(>75 \%)$.

frequency center-of-mass vibrations. The idea comes from using 2-qubit system shown in Figure 4(a), where each path represents a neutron basis state. In this system vibrational noise enters as the phase difference a neutron acquires by passing through different paths due to movement of the interferometer. This noise can be represented in a form of $\sigma_{z}$ Pauli operator. By removing the center blade (Figure 4(b)) we notice that the phase difference acquired by a neutron traveling through paths $|01\rangle$ and $|10\rangle$ due to the vibrational noise will be the same. Thus if we encode the state into logical 


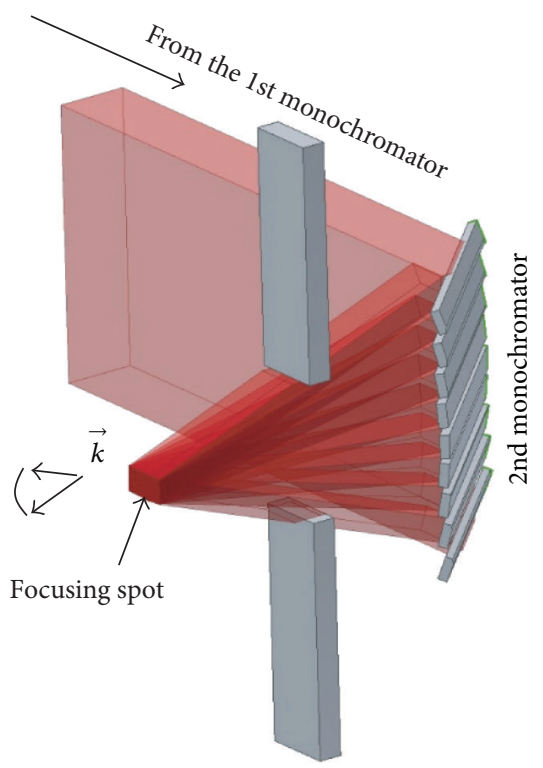

Adjustable slit

(a)
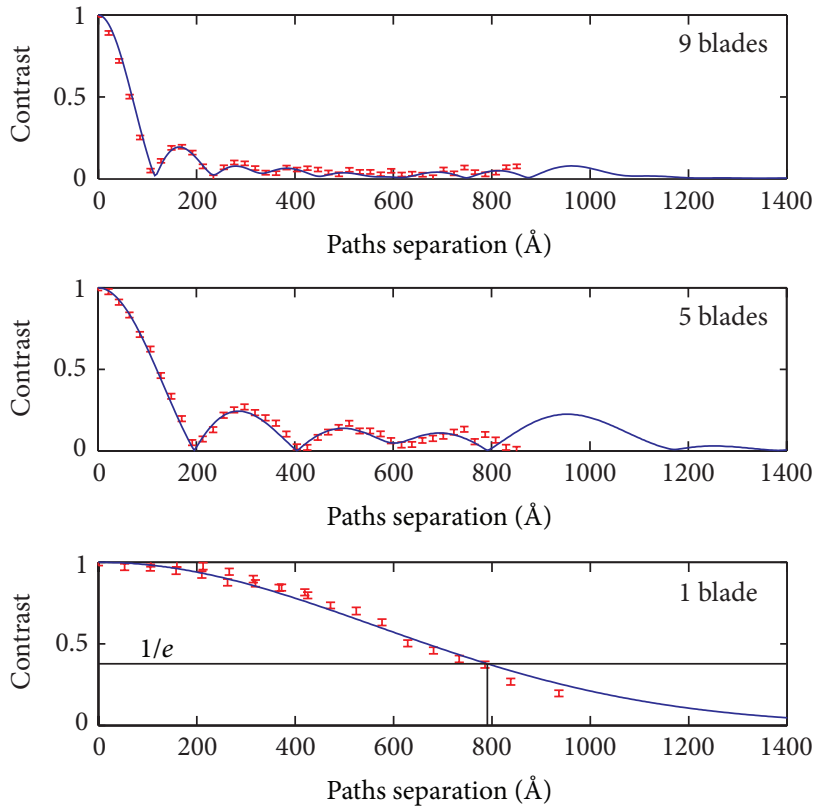

(b)

FIGURE 3: (a) Schematic of the second focusing PG monochromator for shaping the vertical momentum distribution of the neutron beam. By shielding some of the blades of the monochromator we can select different neutron momentum distributions. (b) Contrast versus path separations for different momentum distributions. The top figure is obtained when 9 blades of the 2 nd monochromator are open, the middle figure when 5 middle blades are open, and the bottom figure when only one middle blade is open [14].

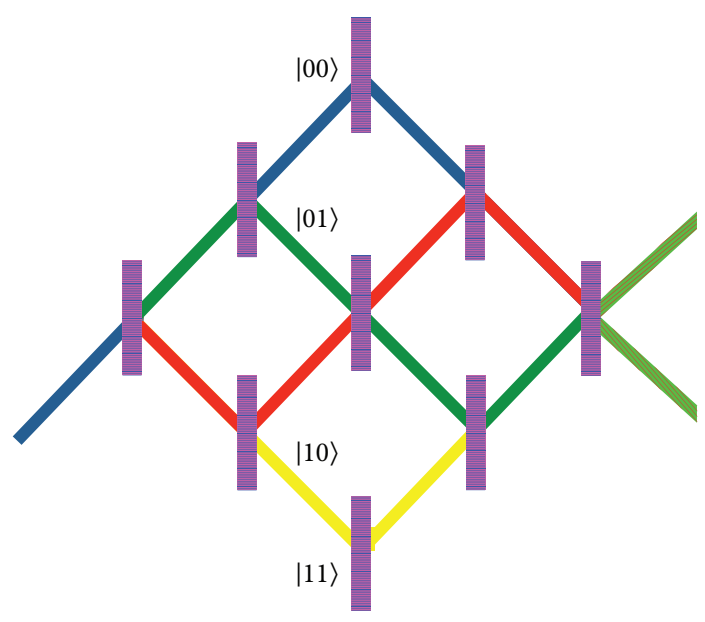

(a)

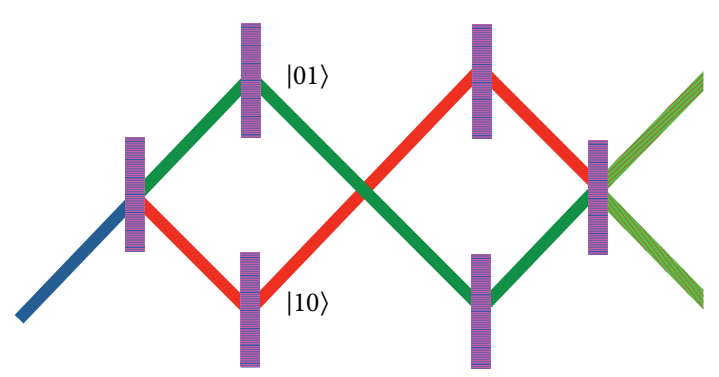

(b)

FIGURE 4: (a) Schematic of the 2-qubit (four paths) interferometer. (b) Schematic of the DFS interferometer.

states $\left|0_{L}\right\rangle=|01\rangle$ and $\left|1_{L}\right\rangle=|10\rangle$ then any state represented in this basis will be protected against this vibrational noise. As a result we came up with a DFS design for neutron interferometer shown in Figure 4(b).

To test our theory we built a 5-blade neutron interferometer [22] to accommodate both the Mach-Zehnder and DFS interferometer designs. This allows us to in situ change between these two designs without disturbing the facility and avoids discrepancies due to different crystal qualities.
Experimental results are shown in Figure 5(a). Here the top and bottom figures show data for the Mach-Zehnder and DFS interferometers, respectively. The red points give the interferogram without applied vibrations while blue points are when an $8 \mathrm{~Hz}$ vibration is applied to the interferometer. Figure 5(a) shows that $8 \mathrm{~Hz}$ vibrations are enough to destroy interference of the Mach-Zehnder interferometer while they do not have much effect on the contrast of the DFS interferometer. Figure 5(b) represents numerical simulation of the 

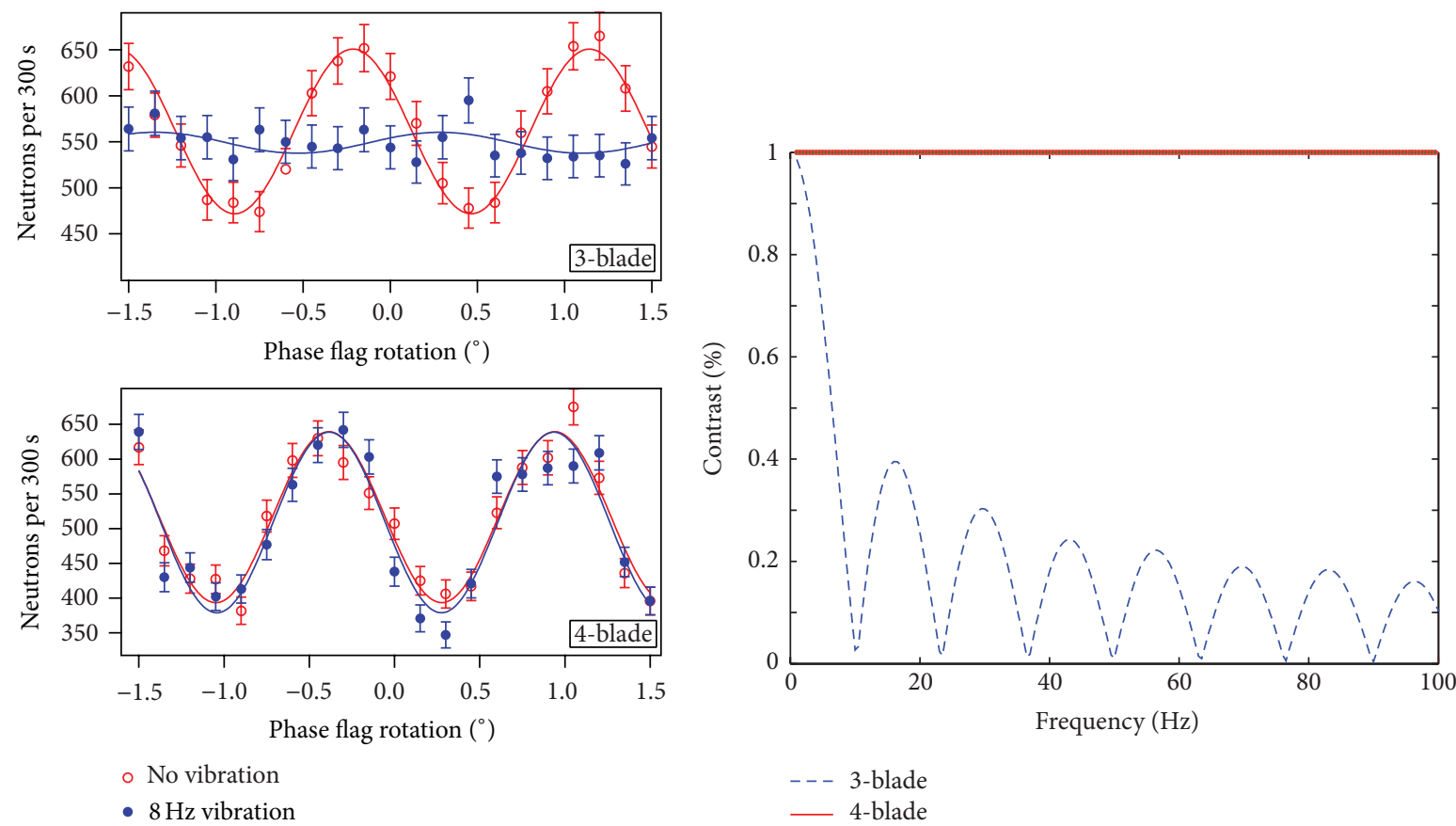

(a)

(b)

FIGURE 5: (a) Comparison data for standard (top) and DFS (bottom) interferometer designs without (red) vibrations and with (blue) 8 Hz applied vibrations. (b) Numerical simulation of standard interferometer (blue) and DFS (red) contrast versus rotational vibrations [22].

contrast for both the Mach-Zehnder (3-blade) and DFS (4blade) setups versus frequency of applied vibrations [23]. These simulations clearly show the advantage of the DFS setup in the presence of vibrational noise.

Another aspect of noise in the neutron interferometer we investigated was the effect of random phase noise on the correlations between the neutron path and neutron spin. This type of noise can arise due to surface imperfections in the interferometer blades which introduce a random phase to neutrons traveling through interferometer, thus reducing the measured contrast of the interferometer when averaged over many neutrons. By entangling the path degree of freedom of the neutrons with the neutron spin we investigated how the phase noise behaves in the presence of a postselected spin measurement on the neutron beam. To quantify the correlations between the spin and path degree of freedom we compared quantum discord and entanglement. Quantum discord was originally proposed as a measure of the "quantumness" of correlations between two quantum systems that is more general than entanglement. It can be thought of as quantifying how much disturbance the measurement of one subsystem of a bipartite quantum system can induce on the other.

To investigate this effect we considered a configuration where we entangled the spin and path degrees of freedom of the neutron by placing a spin-rotator in one path of the interferometer and then observed the output path contrast and spin contrast with and without postselected spin measurements [24]. In the absence of spin measurements, our theoretical calculations found that strong phase noise caused the entanglement to reduce to zero; however the quantum discord remained nonzero. This indicates that nonclassical correlations persist between the spin and path of the neutron and therefore must still be treated as a quantum system. Our calculations predicted, and our experimental results confirmed, that by measuring the Z-component of the neutron spin we could revive spin contrast even in the strong noise case where it would be zero in the absence of spin filtering. Our experimental setup consisted of thin permalloy films deposited on a Si substrate to implement the path-dependent spin rotation, and the spin measurements were implemented using an adiabatic coil to rotate the desired neutron spin state to the Z-basis where spin-up and spin-down neutrons were absorbed and transmitted, respectively, by either Heusler crystals or reflection-mode curved supermirrors.

\section{A New Beam Line}

Following our progress in understanding the effects of noise on the neutron interferometer and our advances in DFS interferometer design, we have decided to build a new beam line which utilizes the DFS interferometer. This beam line would have relaxed restrictions on the low frequency vibration isolation system. A typical optical table should be sufficient to operate a DFS interferometer beam line. This tremendously simplifies the beam line construction and, in principle, should improve neutron fluency with respect to the existing setup. Figure 6 shows a schematic of the existing beam line and outline for the new beam line. 


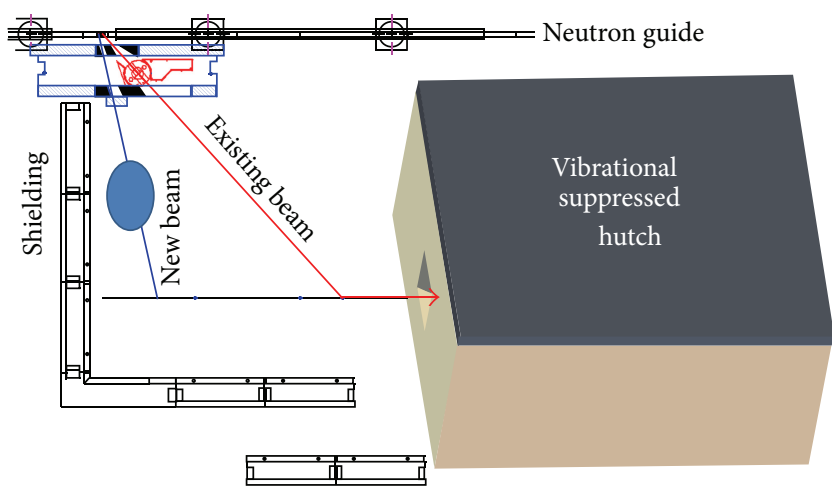

FIGURE 6: Schematic of the existing beam line setup with the hutch and a new beam line for the DFS interferometer.

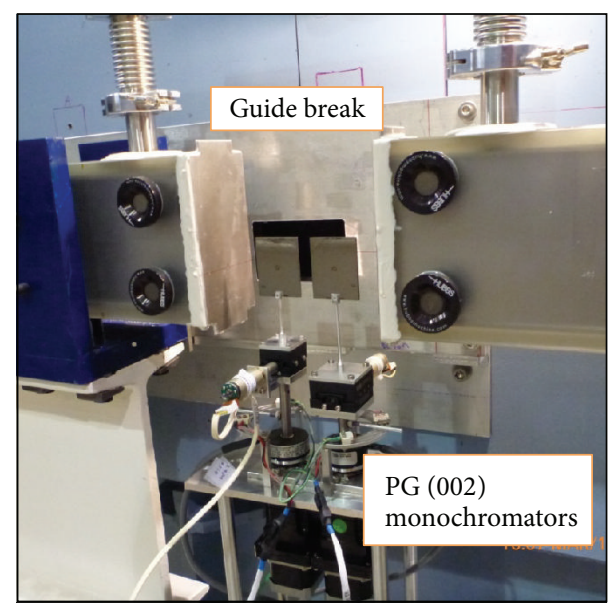

FIGURE 7: A picture of the monochromators for both beam lines inside the guide shielding of the NG7 guide. The crystals are PG crystals with $0.5^{\circ}$ mosaic placed in the NG7 guide break.

We extract neutrons from the NG7 NIST NCNR cold beam guide by Bragg diffraction of PG crystal monochromators. Figure 7 shows the NG7 guide break with two new PG crystal monochromators: one is for the existing beam line and another is for the DFS interferometer beam line. This configuration will allow us to independently control the wavelength of the neutrons of both beam lines.

The current configuration shown in Figure 8 is configured for $\lambda=0.44 \mathrm{~nm}$ neutron wavelength and consists of an optical table (without vibration isolation), a pair of double-V cavity polarizer/analyzer (with greater than $98 \%$ polarization efficiency), and ${ }^{3} \mathrm{He}$-detectors. Due to the high relative count rate at fractional wavelengths $(\lambda / n$, where $n=2,3, \ldots)$ we have installed a Be filter as shown in Figure 8. The beam line is currently under active construction and development.

\section{Conflict of Interests}

The authors declare that there is no conflict of interests regarding the publication of this paper.

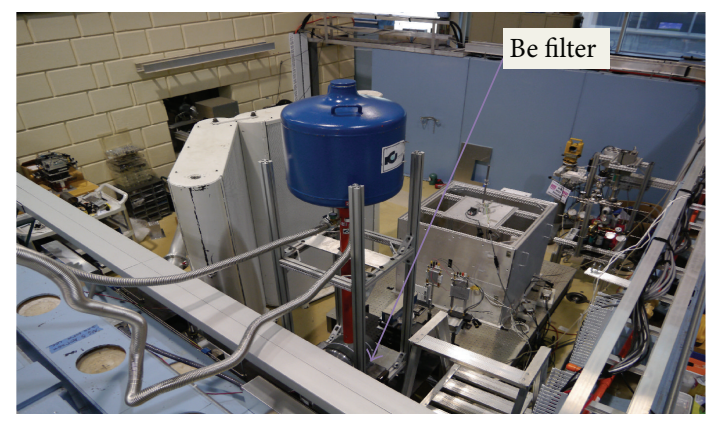

Figure 8: A picture of the new beam line. It consists of a Be filter to remove $\lambda / 2$ and $\lambda / 3$ components, spin polarizer, spin analyzer, adiabatic spin flippers, temperature controlled enclosure for interferometer, and ${ }^{3} \mathrm{He}$-detectors.

\section{Acknowledgments}

This work was supported in part by CREATE, Discovery, NSERC, Industry Canada, CERC, CIFAR, and Ministry of Research and Innovation, Province of Ontario, Canada. Support provided by NIST is also gratefully acknowledged. The authors are grateful to Sam Potts and University of Missouri-Columbia Physics Machine Shop for a wonderful job machining the interferometer and for discussions with $\mathrm{D}$. L. Jacobson, T. Borneman, R. A. Barankov, P. Cappellaro, E. R. Sparks, and G. L. Greene.

\section{References}

[1] H. Rauch, W. Treimer, and U. Bonse, "Test of a single crystal neutron interferometer," Physics Letters A, vol. 47, no. 5, pp. 369371, 1974.

[2] R. Colella, A. W. Overhauser, and S. A. Werner, "Observa tion of gravitationally induced quantum interference," Physical Review Letters, vol. 34, no. 23, pp. 1472-1474, 1975.

[3] J.-L. Staudenmann, S. A. Werner, R. Colella, and A. W. Overhauser, "Gravity and inertia in quantum mechanics," Physical Review A, vol. 21, no. 5, pp. 1419-1438, 1980.

[4] S. A. Werner, H. Kaiser, M. Arif, and R. Clothier, "Neutron interference induced by gravity: new results and interpretations," Physica B+C, vol. 151, no. 1-2, pp. 22-35, 1988.

[5] D. L. Jacobson, S. A. Werner, and H. Rauch, "Spectral modulation and squeezing at high-order neutron interferences," Physical Review A, vol. 49, no. 5, pp. 3196-3200, 1994.

[6] K. C. Littrell, B. E. Allman, and S. A. Werner, "Two-wavelengthdifference measurement of gravitationally induced quantum interference phases," Physical Review A, vol. 56, no. 3, pp. 17671780, 1997.

[7] H. Kaiser, N. L. Armstrong, F. E. Wietfeldt et al., "Gravitationally induced quantum interference using a oating interferometer crystal," Physica B: Condensed Matter, vol. 2, pp. 1384-1387, 2006.

[8] M. Zawisky, J. Springer, and H. Lemmel, "High angular resolution neutron interferometry," Nuclear Instruments and Methods in Physics Research, Section A: Accelerators, Spectrometers, Detectors and Associated Equipment, vol. 634, Supplement 1, pp. S46-S49, 2011. 
[9] G. L. Greene and V. Gudkov, "Neutron interferometric method to provide improved constraints on non-Newtonian gravity at the nanometer scale," Physical Review C, vol. 75, no. 1, Article ID 015501, 2007.

[10] P. Brax, G. Pignol, and D. Roulier, "Probing strongly coupled chameleons with slow neutrons," Physical Review D: Particles, Fields, Gravitation and Cosmology, vol. 88, no. 8, Article ID 083004, 2013.

[11] Y. N. Pokotilovski, "Strongly coupled chameleon fields: possible test with a neutron Lloyd's mirror interferometer," Physics Letters B, vol. 719, no. 4-5, pp. 341-345, 2013.

[12] A. P. Serebrov, P. Geltenbort, O. M. Zherebtsov et al., "Experimental search for long-range forces in neutron scattering via a gravitational spectrometer," Physical Review C, vol. 89, no. 4, Article ID 044002, 2014.

[13] M. Arif, D. E. Brown, G. L. Greene, R. Clothier, and K. Littrell, "Multistage position-stabilized vibration isolation system for neutron interferometry," in Vibration Monitoring and Control, vol. 2264 of Proceedings of SPIE, pp. 20-26, San Diego, Calif, USA, 1994.

[14] D. A. Pushin, M. Arif, M. G. Huber, and D. G. Cory, "Measurements of the vertical coherence length in neutron interferometry," Physical Review Letters, vol. 100, no. 25, Article ID 250404, 2008.

[15] M. G. Huber, M. Arif, T. C. Black et al., "Precision measurement of the n-He3 incoherent scattering length using neutron interferometry," Physical Review Letters, vol. 102, no. 20, Article ID 200401, 2009.

[16] H. Rauch and S. A. Werner, Neutron Interferometry, Oxford University Press, New York, NY, USA, 2000.

[17] U. Bonse and H. Rauch, Neutron Interferometry, Oxford University Press, Oxford, UK, 1979.

[18] H. Kaiser, S. A. Werner, and E. A. George, "Direct measurement of the longitudinal coherence length of a thermal neutron beam," Physical Review Letters, vol. 50, no. 8, pp. 560-563, 1983.

[19] H. Rauch, H. Wölwitsch, H. Kaiser, R. Clothier, and S. A. Werner, "Measurement and characterization of the threedimensional coherence function in neutron interferometry," Physical Review A, vol. 53, no. 2, pp. 902-908, 1996.

[20] "Chapter 4, coherence properties," in Neutron Interferometry, $\mathrm{H}$. Rauch and S. A. Werner, Eds., Oxford University Press, Oxford, UK, 2000.

[21] D. A. Lidar and K. B. Whaley, "Decoherence-free subspaces and subsystems," in Irreversible Quantum Dynamics, F. Benatti and R. Floreanini, Eds., vol. 622 of Lecture Notes in Physics, pp. 83120, Springer, Berlin, Germany, 2003.

[22] D. A. Pushin, M. G. Huber, M. Arif, and D. G. Cory, "Experimental realization of decoherence-free subspace in neutron interferometry," Physical Review Letters, vol. 107, no. 15, Article ID 150401, 2011.

[23] D. A. Pushin, M. Arif, and D. G. Cory, "Decoherence-free neutron interferometry," Physical Review A, vol. 79, no. 5, Article ID 053635, 2009.

[24] C. J. Wood, M. O. Abutaleb, M. G. Huber, M. Arif, D. G. Cory, and D. A. Pushin, "Quantum correlations in a noisy neutron interferometer," Physical Review A, vol. 90, no. 3, Article ID 032315, 2014. 

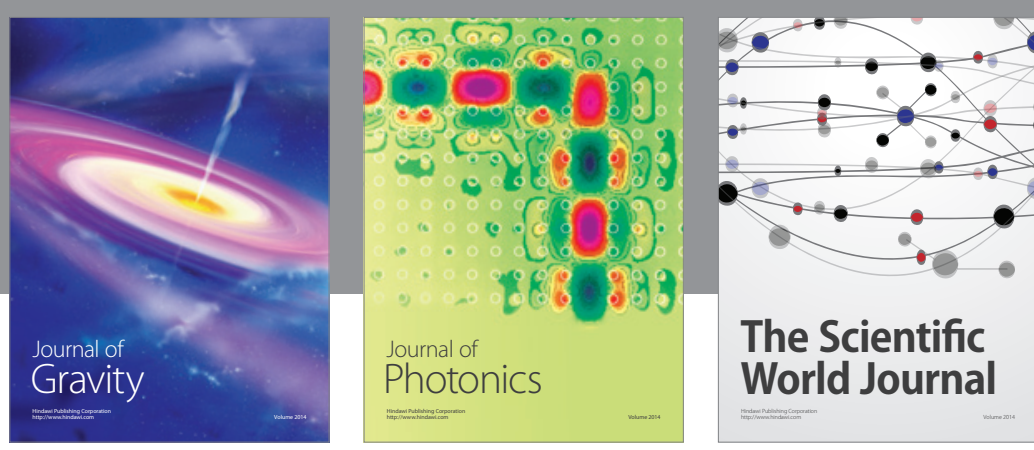

The Scientific World Journal
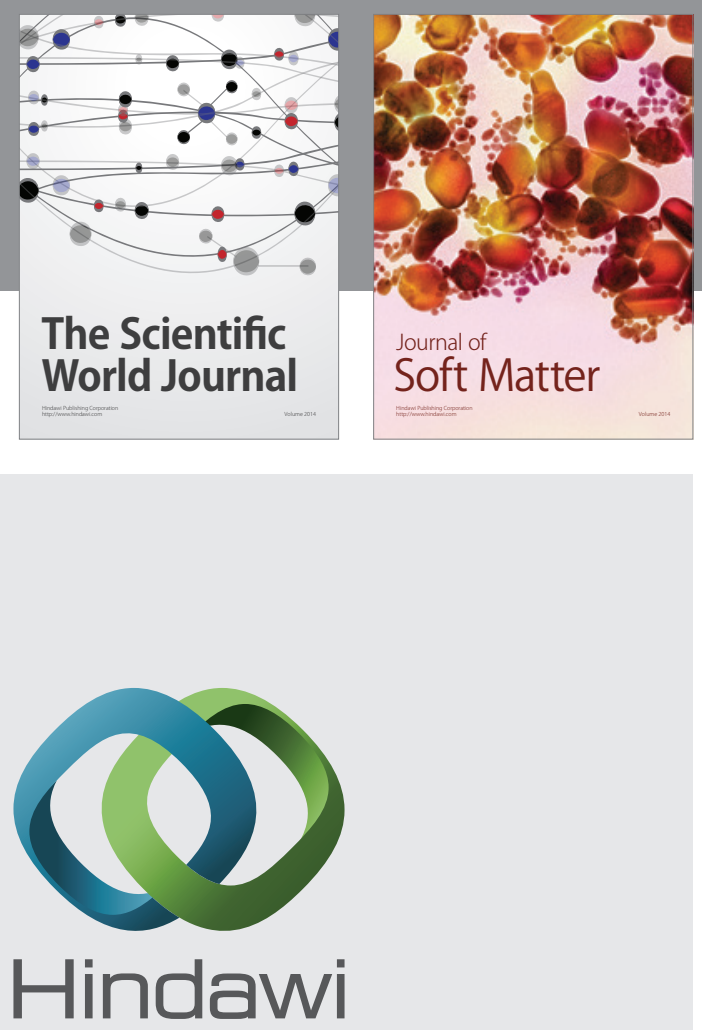

Submit your manuscripts at

http://www.hindawi.com

nternational Journal of

Statistical Mechanics
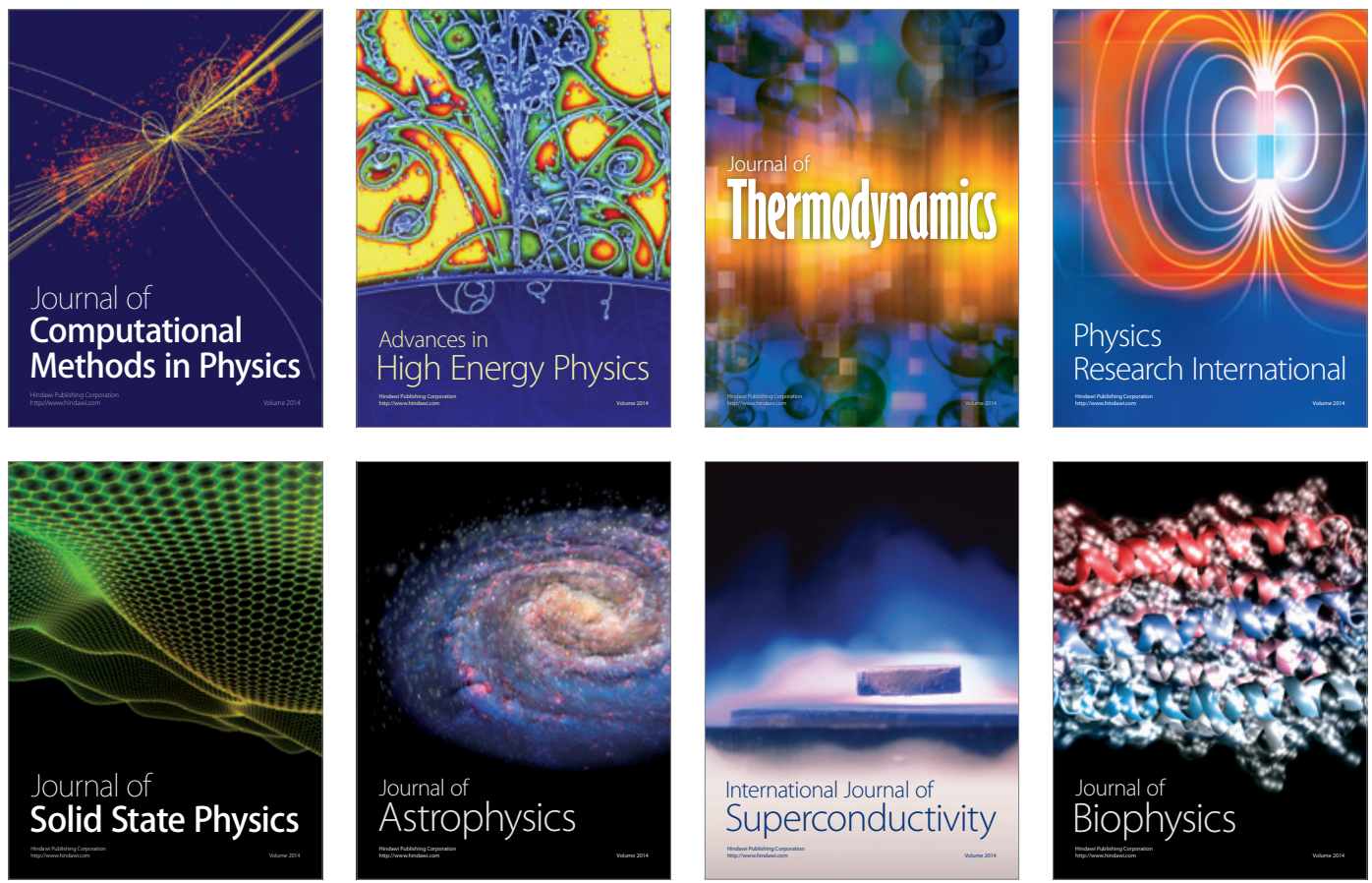
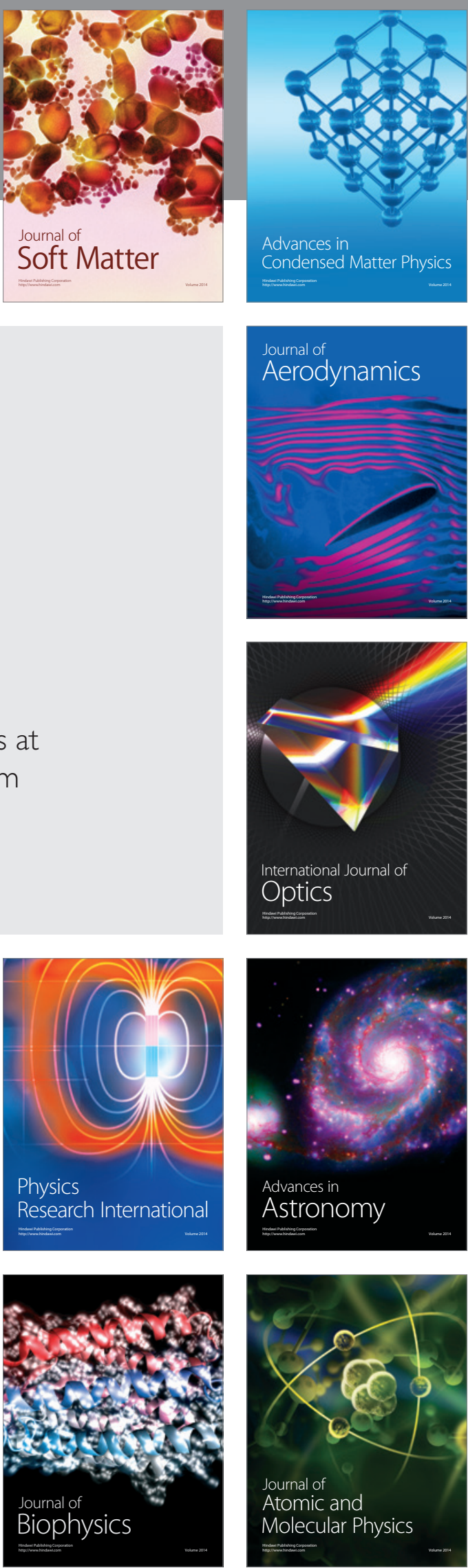